\section{TREATMENT OF SMALL ENCYSTED TUMOURS.}

\section{To the Editor of THE LANCET.}

SIR,-Several ingenious plans having appeared lately in your Journal upon the above subject, each of which appears to me to be more or less tedious, and having extensively practised the following very simple method for a long time past, you will excuse the liberty $I$ have taken in forwarding it to the notice of my professional brethren,- of course it applies to very small tumours, and when situated in parts where the cysts cannot be easily removed with the knife.

I first make a free incision through the tumour, then with a probe slightly bent at its sharp point, effectually remove the whole of its contents ; having done so, freely, apply, with the blunt point of the probe, nitric acid to the whole of the inside of the cyst, sponge the part with cold water, and you have no further trouble; sloughing takes place in two or three days, and the tumour is cured. I have removed a considerable number of small tumours from the eyelids in this manner with the greatest success; of course operating on the inside of the lids, and have never required a second operation on the same tumour. I am, Sir, yours obediently,

$$
\text { F. Page, Surgeon. }
$$

Newmarket, Aug. 17, 1842.

\section{BLACK PITCH IN THE TREATMENT OF H EMORRHOIDS.}

\section{To the Editor of The Lancet.}

Sir,--Should the accompanying remarks upon the use of black pitch in hæmorrhoids merit a place in your valuable Periodical, your insertion of them will oblige yours most respectfully,

\section{Cheetham Hill, near Manchester,} Aug. 17, 1842.

Hæmorrhoids, anatomically speaking, are hypertrophied cellular tissue, in connection with a large number of varicose veins; they are of an indolent or inflammatory character, generally appearing in those persons who live well, and take but little exercise. Out of eighty cases that have come under my notice, sixty occurred in males, and twenty in females; their origin in the latter could be traced to the free use of the pills of the Whitworth doctor, of bone-setting notoriety. These pills are chiefly composed of aloes; in the former the piles were produced in twenty from hard riding; in fifteen their occurrence was attributed to the circumstance of having sat some time upon wet grass; and in the remaining twenty-five, a variety of causes were assigned for the production of hæmorrhoids. The most troublesome symptoms in those patients whose piles resulted from hard riding, were a continual itching about the anus, accompanied with a burning sensation; and to use the expression of the patients themselves, the heat was so intense that it seemed as if " a red-hot iron had been thrust up the fundament." Upon an examination of these persons, a considerable degree of fulness around the verge of the anus was discovered, attended with an inflammatory blush of a leaden hue; the internal surface of the rectum presented to the finger a considerable number of prominences about the size of horse-beans, situated just within the sphincter ani, which were exceedingly tender to the touch, for on gentle pressing them with the point of the finger, the patient complained of a severe lancinating pain; considerable annoyance was experienced from protrusion of the piles after each motion, but no hæmorrhage followed.

The treatment consisted in the application of leeches to the perineum, and in the neighbourhood of the coccyx; for I have witnessed very unpleasant effects from placing leeches upon the verge of the anus when much swollen and inflamed, and more particularly when the inflamed surface has had a dark or leaden appearance; in such cases I have seen small ulcers produced by the leech bites that have required more care and attention to heal than the removal of the piles, and, in one instance, considerable sloughing was the result. I am happy to find that my experience perfectly accords with that of M. Lisfranc, as far as regards the application of leeches to the inflamed parts; and I think with him that the strictest care is necessary in the employment of leeches, and that they ought never to be applied to the anus when in a state of inflammation. After the inflammatory symptoms have subsided, I administered common black pitch, and under its use a speedy cure was effected. In the remaining sixty patients the piles were of an indolent character; in ten, they were attended with a most troublesome discharge of mucus and blood, both before and after a motion. In the remaining fifty, the piles were external ; and in thirty instances they had existed for more than six years, and during that time almost every kind of treatment had been adopted, but without benefit. In one or two of the patients there were evident symptoms of disease of the liver, but in the remainder it seemed that the piles were unaccompanied with any visceral disturbance or organic disease.

Having witnessed the good effects of black pitch in the treatment of piles of an indolent kind, whether attended with hæmorrhage or not, $I$ at once commence with the administration of that substance, combining it with a little blue pill in the two cases already referred to, in whom $I$ apprehended disease of the liver. When given agreeably to the fol. 